Check for updates

Cite this: J. Mater. Chem. B, 2021 9, 7229

Received 4th March 2021

Accepted 5th May 2021

DOI: $10.1039 / \mathrm{d} 1 \mathrm{tb} 00458 \mathrm{a}$

rsc.li/materials-b

\section{Diatom-inspired 2D nitric oxide releasing anti-infective porous nanofrustules}

\author{
Hong Kit Lim, $\dagger^{a}$ Shao Jie Tan, $\dagger^{a}$ Zhuoran Wu, ${ }^{a}$ Boon Chong Ong, (D) \\ Kwan Wee Tan, (D) ${ }^{a}$ Zhili Dong (D) ${ }^{a}$ and Chor Yong Tay (D) *abc
}

Two-dimensional (2D) nanomaterials (NM) have emerged as promising platforms for antibacterial applications. However, the inherent "flatness" of 2D NM often limits the loading of antimicrobial components needed for synergistic bactericidal actions. Here, inspired by the highly ornamented siliceous frustules of diatoms, we prepared 2D ultrathin $(<20 \mathrm{~nm})$ and rigid "nanofrustule" plates via the out-of-plane growth of cetyltrimethylammonium bromide (CTAB) directed silica mesostructures on the surfaces of 2D graphene oxide nanosheets. The nanofrustules were characterized by the presence of mesoporous channels with a pore size of $3 \mathrm{~nm}$ and a high specific surface area of $674 \mathrm{~m}^{2} \mathrm{~g}^{-1}$. $S$-nitrosothiol-modification on the silica surfaces enables the development of a novel anti-infective nitric oxide (NO) releasing NO-nanofrustule system. The cage-like mesoporous silica architecture enabled a controlled and sustainable release of $\mathrm{NO}$ from the NO-nanofrustules under physiological conditions. The NO-nanofrustules displayed broad antibacterial effects against Staphylococcus aureus and Escherichia coli with a minimum inhibitory concentration of $250 \mu \mathrm{g} \mathrm{ml}^{-1}$. Mechanistic studies revealed that the antibacterial property of NO-nanofrustules was attained via a unique "capture-and-release" mode-of-action. The first step entailed the capture of the bacteria by the NO-nanofrustules to form micro-aggregates. This was followed by the release of high levels of NO to the captured bacteria to elicit a potent anti-infective effect. In combination with the lack of cytotoxicity in human dermal cells, the 2D hybrid NO-nanofrustules may be utilized to combat wound infections in clinical settings.

\section{Introduction}

Bacterial pathogenicity is a relentless threat to the global public health. Due to the rapid climate change, globalization and urbanization, there is a significant rise in the number of deaths due to pathogens and infections with major public health repercussions in the past decades. ${ }^{1}$ Based on current trend, infections are projected to kill 10 million people by $2050 .^{2}$ Currently, the use of a single or combinatorial prescription of antibiotics remains the weapon of choice against bacterial infections. Unfortunately, many years of antibiotics misuse have led to the emergence of drug-resistant "superbugs" which has undermined existing anti-infective treatments. ${ }^{3}$ Therefore, the development of novel antibiotic-free strategies to combat pathogenic bacteria is urgently needed.

\footnotetext{
${ }^{a}$ School of Materials Science and Engineering, Nanyang Technological University, 50 Nanyang Avenue, Singapore 639798, Singapore.E-mail: cytay@ntu.edu.sg

${ }^{b}$ School of Biological Sciences, Nanyang Technological University, 60 Nanyang Drive, Singapore 637551, Singapore

${ }^{c}$ Environmental Chemistry and Materials Centre, Nanyang Environment \& Water Research Institute, 1 Cleantech Loop, CleanTech One, Singapore 637141, Singapore

$\dagger$ These authors contributed equally to this work.
}

In the last two decades, numerous classes of nanomaterials such as 0D nanoparticles (NPs), ${ }^{4}$ 1D nanorods ${ }^{5}$ and more recently, 2D planar materials ${ }^{6,7}$ with intrinsic antimicrobial activities have been explored extensively to combat bacterial infections. Compared to antibiotics, the use of nanomaterials is less likely to elicit antimicrobial resistance due to their multiple simultaneous modi operandi. ${ }^{8}$ Owing to the ease of synthesis, tunable size, tailorable chemistries, 2D nanomaterials such as graphene oxide (GO), ${ }^{9}$ reduced graphene oxide (rGO), ${ }^{10}$ transition metal dichalcogenides ${ }^{11}$ (TMD) and MXenes $^{12}$ have emerged as promising novel anti-infective candidates. 2D nanomaterials were reported to elicit potent antimicrobial effects through physicochemical means. For instance, the high surface area and reactivity of $2 \mathrm{D}$ nanomaterials can exert chemical pressure via overproduction of intracellular reactive oxygen species (ROS) through electron transfer ${ }^{13}$ or material surface imperfections, ${ }^{14}$ as well as physical disruption of the bacteria cell membrane. ${ }^{9}$

However, 2D antimicrobial nanomaterials are not omnipotent; the clinical adoption of $2 \mathrm{D}$ nanomaterials is currently curtailed due to their toxic effects in mammalian cells when used at bactericidal concentrations. ${ }^{15}$ Furthermore, if we solely capitalize on the NP properties such as shape, surface charge 
and size for antibacterial activity, various optimizations are required to facilitate the cell membrane penetration ${ }^{16}$ and/or uptake. ${ }^{17}$ Bacterial uptake of NPs can be difficult especially when dealing with a biofilm. ${ }^{18}$ At the same time, most of the $2 \mathrm{D}$ nanomaterials tend to self-aggregate in biological milieu due to the "plate-plate DLVO interaction", 19 thereby further reducing their antibacterial efficacy as a result of reduced bacteria uptake $^{20}$ and may even confer resistance. ${ }^{21}$ Therefore, many studies in this field strive to incorporate other active antimicrobial components, such as peptides, ${ }^{22}$ drugs $^{23,24}$ or small molecules $^{25}$ to the $2 \mathrm{D}$ nanomaterials, aiming for synergistic bacteria-killing effects.

Nitric oxide (NO) is a diatomic, free-radical gas that is naturally produced in the body and plays a major role in innate immunity. In response to an infection, NO is produced in large quantities by macrophages via the NO synthase isoforms (iNOS/ NOS2) that spontaneously react with $\mathrm{O}^{-}$containing superoxides to generate a variety of antimicrobial molecules in the form of reactive nitrogen species such as such as dinitrogen trioxide $\left(\mathrm{N}_{2} \mathrm{O}_{3}\right)$, nitroxyl ( $\left.\mathrm{HNO}\right)$ and peroxynitrite $\left(\mathrm{ONOO}^{-}\right){ }^{26}$ These powerful oxidants can induce irreparable damage to bacterial proteins through nitrosation of reactive thiols and amines, as well as DNA materials. ${ }^{27}$ NO displays non-specific broad-spectrum antimicrobial activity against a wide range of Gram-positive and Gram-negative bacteria such as Pseudomonas aeruginosa (P. aeruginosa), ${ }^{28}$ Staphylococcus aureus $(S$. aureus $),{ }^{29}$ Escherichia coli (E. coli), ${ }^{30}$ Acinetobacter baumannii (A. baumannii), ${ }^{31}$ Staphylococcus epidermidis (S. epidermidis), ${ }^{32}$ and even the Methicillin-resistant Staphylococcus aureus (MRSA). ${ }^{33}$ NO passes through most physiological barriers easily at the targeted site for effective antimicrobial action. ${ }^{34}$ As such, the choice of a planar structure to serve as a "mothership" for NO delivery offers a distinctive advantage as this type of structure enables greater interaction with bacteria before releasing the high NO payload.

In this study, we report the development of a novel NO releasing $2 \mathrm{D}$ hierarchical porous nanocomposite that is inspired by the bio-silicification process and the ultrastructured "anatomy" of the diatom siliceous cell shells, also called frustules. The process of bio-silicification entails the precise integration of silica materials into an organic matrix to form nanoscale composites with exquisite morphologies. ${ }^{35}$
Herein, GO was used as the organic template for the growth of surfactant-directed silica to create a mesoporous heterostructure 2D nanomaterial that will enable us to maximize the loading capacity of the pharmacological active $S$-nitrosothiol (SNO) moiety as an NO source. Because the resultant silica architecture emulates the highly ornamented silica frustules of diatoms, we term this class of porous $2 \mathrm{D}$ nanomaterials as $2 \mathrm{D}$ "nanofrustule" plates. ${ }^{36}$ The physiochemical properties such as nanoscale surface topography, porosity and NO loading capacity were systematically characterized. As an antimicrobial agent, the design and working principle of nitrosylated nanofrustule (NO-nanofrustule) are premised on the "capture and release" concept - it is envisioned that once the NOnanofrustules have successfully docked onto the surface of the bacterial wall, release and diffusion of cell wall-permeable NO into the target cells can occur to exert a potent bactericidal effect. In contrast to the GO based antimicrobial agents, the presence of the mesoporous silica bilayer confers rigidity and structural stability to the nanofrustules, ensuring maximal presentation of the material surface to seek out and capture the bacteria. $^{37}$ In addition, unlike conventional nanomedicine strategies, ${ }^{38}$ the bacteria-killing effect of nanofrustules does not require active nanomaterials uptake by the microorganisms. As a proof-of-concept, the anti-microbial activity of NOnanofrustules was evaluated in $S$. aureus and E. coli. Furthermore, the biocompatibility of NO-nanofrustules was also validated with keratinocytes and dermal fibroblasts of human origin.

\section{Results and discussion}

Fig. 1 shows the synthesis scheme of the nanofrustules. Graphene nanopowder with an average planar size of $500 \mathrm{~nm}$ to $2 \mu \mathrm{m}$ range was chosen as the base template for oxidation, whereby the previously developed Preformed Acidic Oxidizing Medium (PAOM) method was used to produce highly oxidized GO sheets. ${ }^{39}$ Afterwards, the GO sheets were subjected to the modified Stöber method involving co-condensation of two different silica sources, i.e., TEOS and MPTMS, to form the thiol-functionalized ( $\mathrm{SH}$ ) nanofrustules with the mesoporous silica layers covering the top and bottom faces of the GO sheet.

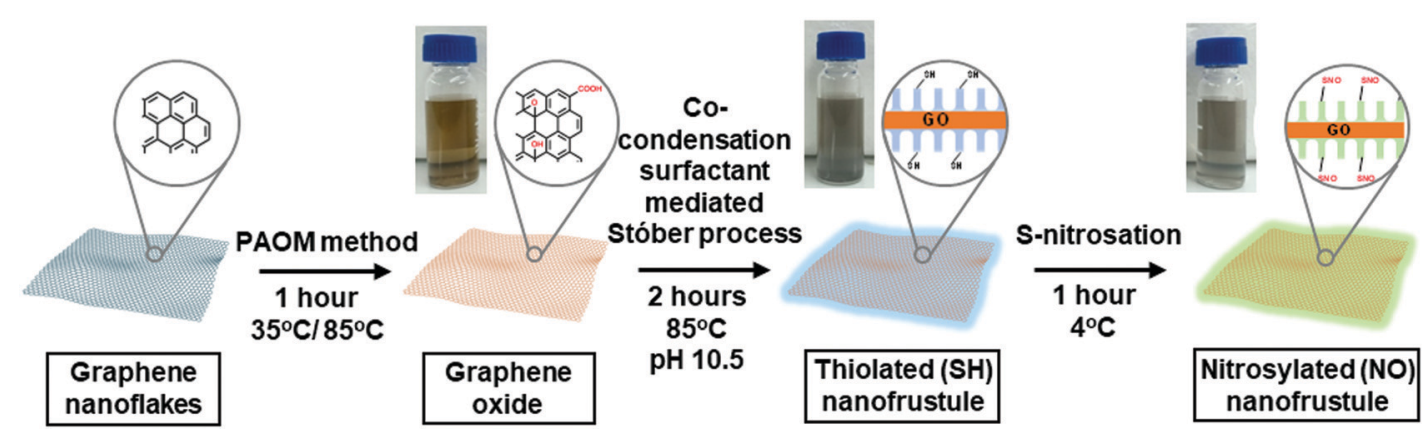

Fig. 1 Schematic diagram showing the synthesis scheme of NO-nanofrustules starting from graphene nanoflakes. Photographic inserts show the color of homogeneous aqueous dispersions of $\mathrm{GO}, \mathrm{SH}$-nanofrustules and $\mathrm{NO}$-nanofrustules. 
The final ratio of the two silica sources was optimized in accordance to an earlier study. ${ }^{40}$ Finally, the final NO-loaded nanofrustules were formed via $S$-nitrosation which involves the formation of the S-NO bond from the thiol group. Both SH- and NO-functionalized nanofrustules displayed good dispersibility in water, forming a uniform suspension after 1-2 minutes of ultrasonication.

Fig. 2a shows the representative SEM image of Coscinodiscus sp., a genus of centric diatom that is characterized by a distinct honeycomb-shaped array of pores covering the valve surface that is believed to maximize contact with the extracellular environment for efficient nutrient and gas exchange. ${ }^{41}$ In the case of the synthetic NO-nanofrustule, similar periodic nanoscale $2 \mathrm{D}$ pore arrays were grown on the surfaces of the GO template. The as-synthesized CTAB-directed mesoporous silica structure had an average pore size of around $3 \mathrm{~nm}$ as shown in Fig. 2b. This contrasts with the TEM image of GO nanosheets which depicts a smooth and non-textured surface (see Fig. S1 in the ESI $\dagger$ ). The mesoporous characteristics of the $\mathrm{SH}$ nanofrustules were confirmed in nitrogen sorption measurements, displaying a Type IV isotherm with a hysteresis loop (see Fig. S2 in the ESI $\dagger$ ). BET specific surface area of nanofrustules obtained from the nitrogen sorption analysis was $674 \mathrm{~m}^{2} \mathrm{~g}^{-1}$, which is significantly higher compared to dried graphite oxide (20-40 $\left.\mathrm{m}^{2} \mathrm{~g}^{-1}\right)^{42}$ and mesoporous silica nanoparticles $\left(18-630 \mathrm{~m}^{2} \mathrm{~g}^{-1}\right){ }^{43}$ Based on the TEM images of the synthesized products, the nanofrustules not only appeared to be significantly thicker than the GO nanosheets after silicification, but also smaller in terms of the average planar size (see Fig. S1 in the ESI $\dagger$ ). Worm-like mesopores were also observed on the
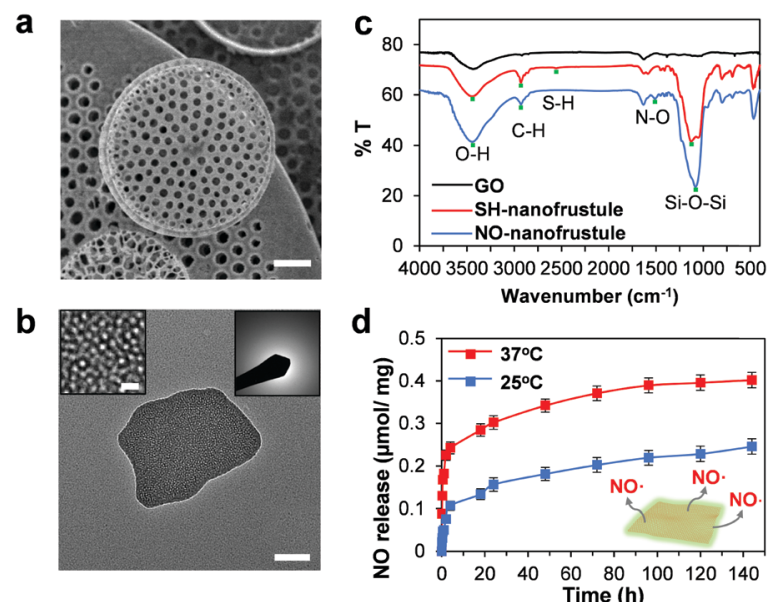

Fig. 2 Structural and physicochemical characterization of nanofrustules. (a) FESEM image of the Coscinodiscus sp. diatom frustule, lying on top of other frustules. Scale bar $=5 \mu \mathrm{m}$. (b) Representative bright field TEM image of a single NO-nanofrustule. Scale bar $=100 \mathrm{~nm}$. Left inset indicates mesopores (bright spots) on the surface of NO-nanofrustules arranged in worm-like fashion, scale bar $=5 \mathrm{~nm}$. Right inset indicates the absence of an observable electron diffraction pattern, suggesting the amorphous nature of the silica layers. (c) FTIR spectroscopic results for GO and the nanofrustules with important peaks labelled. (d) NO release profile for NOnanofrustules in PBS at body and room temperatures, showing ability of controlling the NO release by heat. surface of the nanofrustules, which is in agreement with the mesoporous structure obtained using a similar synthesis method. ${ }^{44}$ The pore size of the mesopores was around $3 \mathrm{~nm}$, when measured using BJH analysis (see Fig. S3 in the ESI $\dagger$ ).

Shown in Fig. 2c are the FITR spectra of the GO, as well as thiolated ( $\mathrm{SH}$ ) and nitrosylated (NO) nanofrustules. After conjugation of silica and thiol functional groups, the characteristic peaks at approximately $451 \mathrm{~cm}^{-1}$ ( $\mathrm{Si}-\mathrm{O}-\mathrm{Si}$ asymmetric stretch), $564 \mathrm{~cm}^{-1}$ (C-S stretch), $694 \mathrm{~cm}^{-1}$ (C-Si stretch), $1128 \mathrm{~cm}^{-1}$ (Si-O-Si asymmetric stretch), $1450 \mathrm{~cm}^{-1}(\mathrm{C}-\mathrm{H}$ bending in methyl group), $2560 \mathrm{~cm}^{-1}$ (S-H stretch) and $2930 \mathrm{~cm}^{-1}(\mathrm{C}-\mathrm{H}$ stretch in methyl group) appeared in the nanofrustules samples while they were absent in GO. This indicates that the thiolfunctionalized silica was successfully conjugated onto the GO template. Successful thiol conjugation in the nanofrustules was further validated by the quantitative Ellman assay, where we found that the free thiol group concentration in the $\mathrm{SH}$ nanofrustules was $0.41 \pm 0.08 \mu \mathrm{mol} \mathrm{mg}{ }^{-1}$. The reduction in wavenumber for the peaks at $1058 \mathrm{~cm}^{-1}(\mathrm{C}=\mathrm{O}$ stretch $)$ and $1384 \mathrm{~cm}^{-1}$ (edge $\mathrm{C}-\mathrm{C}$ vibration) after conjugation indicates that the $\mathrm{C}=\mathrm{O}$ and edge $\mathrm{C}$ bonds in the graphene $\mathrm{sp}^{2}$ structure became weaker. This may be due to the added silica layer covering both sides of the GO nanosheet, therefore reducing the intensity of $\pi-\pi$ stacking between two graphene layers of the nanofrustules. In addition, the characteristic peak at approximately $1520 \mathrm{~cm}^{-1}$ (N-O stretch) for NO-nanofrustule samples confirmed the presence of the nitroso functional group.

Next, to elucidate the NO releasing capability of the NOnanofrustules, the amount of NO liberated in phosphate buffer solution (PBS) was measured using the Griess reagent method. Fig. $2 \mathrm{~d}$ shows the time-dependent release of $\mathrm{NO}$ at $25{ }^{\circ} \mathrm{C}$ and $37{ }^{\circ} \mathrm{C}$. In both experimental groups, we observed an initial burst release of NO in the first 2 hours, followed by a near zero-order sustained release over a period of 4 days. The half-life of NO release from NO-nanofrustules was approximately 1.5 hours. The initial burst NO release may be caused by the spontaneous nature of the S-NO bond cleavage, accompanied by an increase in the local temperature as the suspension equilibrates to $37{ }^{\circ} \mathrm{C}$, which is consistent with the literature. ${ }^{40}$ The prolonged NO release may be attributed to the "cage effect" of the mesoporous silica structure of NO-nanofrustules, whereby after the homolytic cleavage of the S-NO bond, the resultant thiyl radical and NO radical tend to recombine in the tightly confined spaces of the mesopores. ${ }^{45}$ The release profile could be modelled using the Korsmeyer-Peppas model for drug release, ${ }^{46}$ whereby a best-fit straight line could be obtained by plotting log of cumulative percentage of NO release $v s$. log time. The gradient of the best fit line is $m=0.2891$ with a correlation coefficient of 0.9797 (see Fig. S3 in the ESI $\dagger$ ). In this model, a value of $m$ equal to or smaller than 0.5 describes a Fickian diffusion release mechanism. ${ }^{47}$ However, the cumulative amount of NO released at the end of 4 days in $37{ }^{\circ} \mathrm{C}$ $\left(0.41 \mu \mathrm{mol} \mathrm{mg} \mathrm{m}^{-1}\right)$ was significantly higher than the $25{ }^{\circ} \mathrm{C}$ (0.25 $\mu \mathrm{mol} \mathrm{mg}^{-1}$ ) experimental set, suggesting that the NO-nanofrustule has moderate thermal stability and moderate S-NO bond energy. 
a
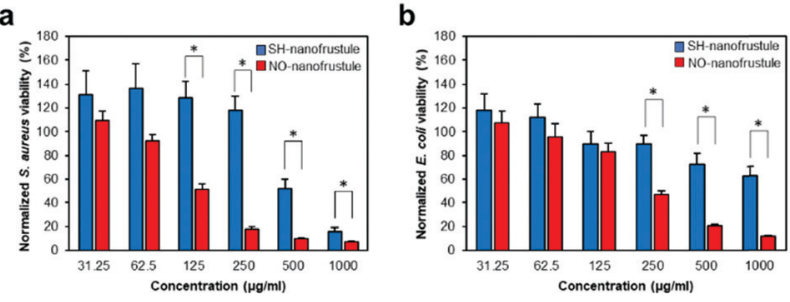

C

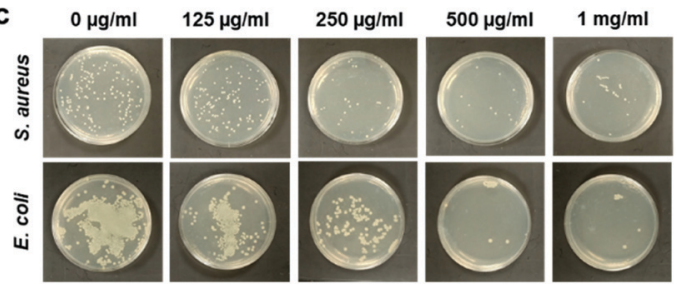

Fig. 3 Antibacterial broth microdilution assay results for (a) S. aureus and (b) E. coli. (c) Agar regrowth results after treatment with different concentrations of NO-nanofrustules for 2 hours at $37^{\circ} \mathrm{C}$. Data represent mean \pm standard deviation of the percentage viability of three replicates normalized to the untreated control. Experiments were triplicated. * denotes statistical significance between two sample groups, where $p<0.05$

Taking into consideration the half-life of the NO release i.e., 1.5 hours, we conducted the antibacterial broth dilution assay by exposing it to 0.0625 to $1 \mathrm{mg} \mathrm{ml}^{-1}$, in 2 -fold increments, of NO-nanofrustules for 2 hours. The corresponding results from the assay are shown in Fig. $3 \mathrm{a}$ and b. To decouple the antibacterial contributions from NO from the effects of the $2 \mathrm{D}$ nanostructure, SH-nanofrustules were used as an internal control. As expected, both 2D nanomaterials displayed a dosedependent killing profile against both types of the bacteria. This observation is consistent with numerous reports on the innate anti-microbial properties of $2 \mathrm{D}$ nanomaterials. However, compared to SH-nanofrustules, NO-nanofrustules displayed a significant enhancement in the antibacterial efficacy, with a half minimum inhibitory concentration (MIC50) of $250 \mu \mathrm{g} \mathrm{ml}^{-1}$ in both the $S$. aureus and $E$. coli groups. In contrast, the bacteria killing efficacy of SH-nanofrustules was clearly lower as indicated by a MIC50 of $1 \mathrm{mg} \mathrm{ml}^{-1}$. Furthermore, the minimum bactericidal concentration (MBC) of NO-nanofrustules, whereby more than $99 \%$ of bacteria were killed, was determined to be $4 \mathrm{mg} \mathrm{ml}^{-1}$ (data not shown). To further demonstrate the antibacterial potential of NO-nanofrustules, a diluted fraction (1/10000) of the surviving bacteria following the 2 hours treatment of various concentrations of the bacteria were plated on agar plates for 24 hours. Fig. 3c shows that the number of viable bacteria colonies of both $S$. aureus and $E$. coli groups decreased in a dose-dependent manner, until $1 \mathrm{mg} \mathrm{ml} \mathrm{m}^{-1}$, whereby agar regrowth of the bacteria was minimum. Therefore, the results show that the NOnanofrustules have potent non-specific antibacterial activity, in comparison to their predecessor SH-nanofrustules with mild antibacterial activity.

Comparing the antibacterial activity of NO-nanofrustules with other similar published materials in retrospect, the bestperforming NO-releasing silica nanorods by Yuan's group
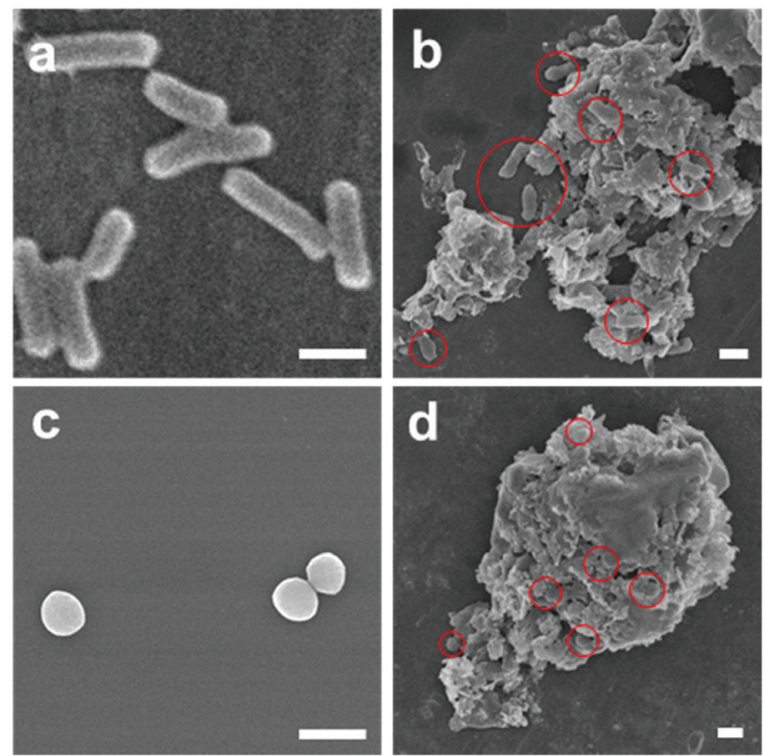

Fig. 4 FESEM images depicting (a) untreated E. coli; (b) treated E. coli; (c) untreated S. aureus and (d) treated S. aureus. The treated bacterial samples were exposed to NO-nanofrustules at $250 \mu \mathrm{g} \mathrm{ml}^{-1}$ for 2 hours. Aggregated clumps consisting of distorted bacteria (marked by red circles) and stacks of the nanoflakes were formed, regardless of the Gram-type of the bacteria. Scale bar $=1 \mu \mathrm{m}$.

required at least $70 \mu \mathrm{M}$ of total NO dose to kill $S$. aureus, ${ }^{48}$ while the NO-nanofrustules required less than $50 \mu \mathrm{M}$ of total released NO to kill S. aureus. Similarly, Grommersch's group who used real diatom frustules bio-templated with $S$-nitrosothiols achieved good antibacterial efficacy at relatively high concentrations, i.e., in the order of 1-10 $\mathrm{mg} \mathrm{ml}^{-1}{ }^{49}$

The high antibacterial efficacy of NO-nanofrustules sets the stage for further investigation in the mechanism of bacteria killing by this material. We first hypothesize that due to the high surface area of NO-nanofrustules, it is an excellent platform to trap or interact with the bacteria in the proximity until the localized NO flux is large enough to kill them. FESEM images of the bacteria colonies treated with the NOnanofrustules confirmed this hypothesis. Fig. 4a and c show the morphology of normal $E$. coli and $S$. aureus under SEM, respectively, prior to treatment. Fig. $4 \mathrm{~b}$ and d show that after treatment with $250 \mu \mathrm{g} \mathrm{m} \mathrm{m}^{-1}$ of NO-nanofrustules, they form micrometer-scale nanomaterial-bacteria aggregates. As a result, almost all the bacteria were observed to interact extensively with the nanomaterials, in such a way that the local NO flux can quickly increase to a lethal level. In addition, some indistinguishable features, other than the flake-like nanofrustules or distorted bacteria, were observed, possibly consisting of the extracellular polymeric matrix (EPS) secreted by the bacteria as a protective attempt against stress. ${ }^{50}$

To visualize how the bacteria were killed by NOnanofrustules, we next conducted a BacLight ${ }^{\mathrm{TM}}$ bacterial viability fluorescence assay on the mixture of NO-nanofrustules and bacteria. In Fig. 5a and b, the green and red spots represent the state of bacteria as alive and dead, respectively. The clump appeared as an overlap of slightly brighter green or red spots, 


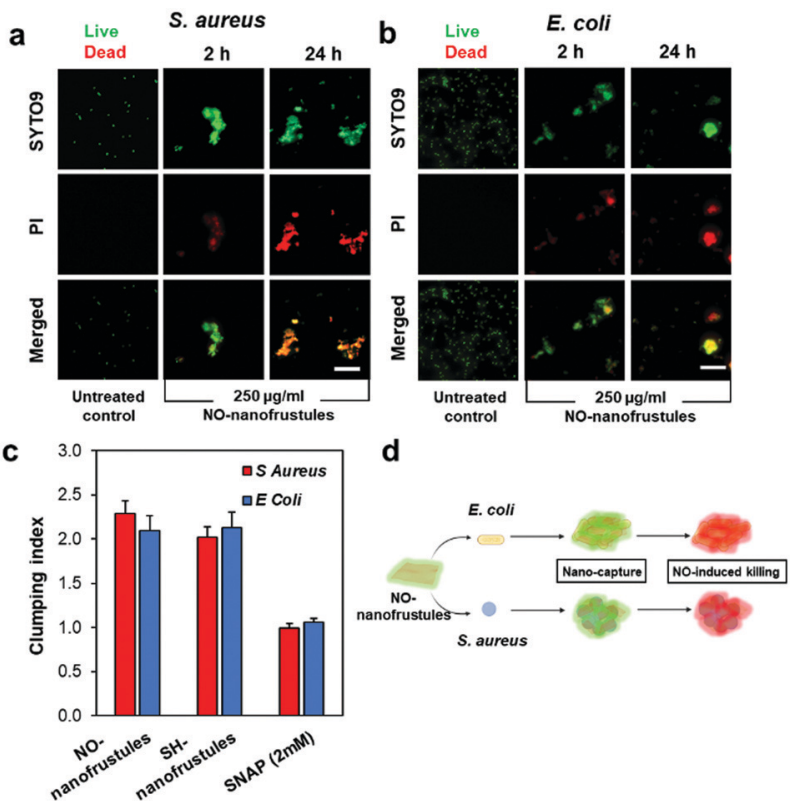

Fig. 5 Representative BacLight ${ }^{\mathrm{TM}}$ fluorescence micrographs (SYTO9 and $\mathrm{Pl}$ staining) of (a) S. aureus and (b) E. coli as a function of treatment (with NO- or SH-nanofrustules, untreated) and incubation times (2 hours or 24 hours). Live bacteria cells are labelled green, while dead bacteria cells are labelled red. Scale bar $=10 \mu \mathrm{m}$. (c) Computed clumping index of $S$. aureus and $E$. coli treated with NO-, SH-nanofrustules or 2 mM of SNAP, normalized to untreated bacteria. Values represent a mean \pm standard deviation of 30 independently resolved particles in ImageJ. (d) Schematic illustrating the mechanism-of-action of NO-nanofrustules.

representing the bacteria and the aggregated layered mass of the NO-nanofrustules. This observation suggests that most of the bacteria trapped within the clumps were dead. Compared to the images at 2 hours post-treatment, the images at 24 hours post-treatment show that the bacteria were killed in a much greater extent in the NO-nanofrustules treated samples. In contrast, the untreated control displayed mainly green (live) individual free-floating bacteria.

Based on the analysis of the fluorescence BacLight ${ }^{\mathrm{TM}}$ assay images, the extent of aggregation of the observed aggregated clumps can be quantified by defining a term called clumping index (CI). The CI is defined as the fraction of observable fluorescent area with a maximum Feret diameter greater than 0.015. As shown in Fig. 5c, the bacterial samples treated with both SH-nanofrustules and NO-nanofrustules showed similar CIs, both values of which were higher than those of the untreated and samples treated with $2 \mathrm{mM}$ of $S$-nitroso- $n$ acetylpenicillamine (SNAP), a small molecular donor of NO and a widely recognized antimicrobial. ${ }^{51}$ No significant difference was found between $S$. aureus and E. coli in terms of CI, indicating that the formation of aggregated clumps is nonspecific to the Gram type or the membrane structure of the bacteria.

Taken together, our findings suggest a unique two-step mechanism of action of NO-nanofrustules. The first step, illustrated in Fig. 5d, involves the tendency of bacteria to adsorb to the nanofrustule surface, together with the size-dependent "canvas effect" which helped to capture and wrap surrounding bacteria forming clumps. This is then followed by subsequent killing of the trapped bacteria due to the high NO flux to elicit a potent bactericidal effect. This clump-forming aggregation process is most likely to be thermodynamically and chemically driven. The bacteria were able to adsorb spontaneously to the nanofrustules due to the porous architecture which provides sufficient interactive anchorage points at the bacteria-nanomaterial interface, which resembles the initial step of biofilm formation. However, in our case, instead of being able to propagate healthily on the surface, the adsorption process is clearly an act of suicide for the bacteria due to the high NO flux on the nanofrustule surface. Our approach is markedly different from using conventional antibacterial NO-releasing nanoparticles, which rely on the bacteria as the anchorage point and subsequent bacterial uptake. $^{52}$ Notably, other types of $2 \mathrm{D}$ nanomaterials such as GO, ${ }^{53}$ $\mathrm{MoS}_{2}{ }^{6}$ and MXenes ${ }^{12}$ with a similar lateral size range also demonstrate the specialty of wrapping and capturing bacteria. Further evidence also points to a van der Waals force dominated interaction between amorphous colloidal silica nanoparticles and the dioleoyl phosphatidylcholine (a component of biomembranes) monolayer, independent of the particle size, $\mathrm{pH}$, and the ionic strength of the solution, ${ }^{54}$ hence ruling out the possibility of electrostatic nature of the interaction. Furthermore, a silica substrate with a nanostructured rough surface or porous surface has higher adhesion force with bacteria compared to the non-porous surface, as quantified by surface morphometry. ${ }^{55,56}$ As for the observed shape distortion in both types of bacteria, because GO sheets with lateral sizes of more than $1 \mu \mathrm{m}$ are found to wrap and trap bacteria, causing bacterial cells to be intertwined together alongside a perturbation to the bacterial membrane potential, ${ }^{57}$ the nanofrustules with a flake-like structure may have inherited this specialty from their GO predecessor. Another possible explanation for this aggregation process is the previously reported "phase-to-size transformation" as seen in thermal responsive polymer brush-conjugated $\mathrm{Zn}$-doped carbon nanosheets, involving a thermal-driven hydrophilic-to-hydrophobic switching of the nanosheet surface. This transformation was found to improve trapping and wrapping-induced local bacteria eradication. However, the transformation of our study is not as drastic because the attempt to alter the hydrophilicity state using external electromagnetic radiation such as NIR has not been employed in this study.

Biocompatibility must be established for the exploitation of NO-nanofrustules to combat infections. We first examined the viability of NO-nanofrustules treated human dermal fibroblasts (HDF) and human keratinocytes (HaCaT) using the Calcein AM/ PI Live/Dead staining assay. In Fig. 6a, the assay highlighted that when exposed to NO-nanofrustules at the MIC50 $(250 \mu \mathrm{g}$ $\mathrm{ml}^{-1}$ ) for 24 hours, majority of the cells remained viable (green), while the occurrence of PI-stained cells (red) was negligible. Furthermore, the nanomaterials treated cells retained their characteristic cellular morphologies of epithelial cells (HaCaT) and fibroblasts (HDF), suggesting that those cells remained healthy. 
a

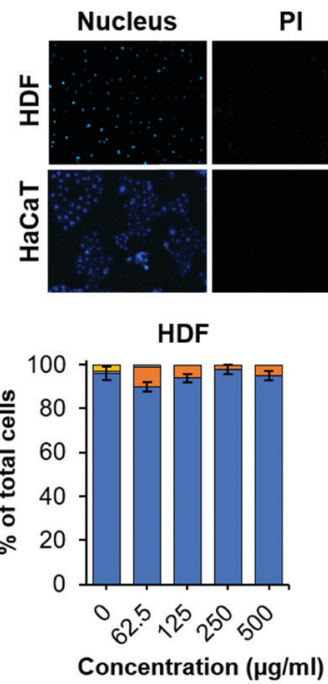

Calcein AM Merged

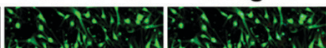



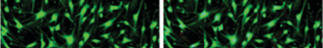

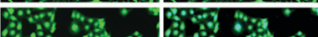
3 释 C HaCaT

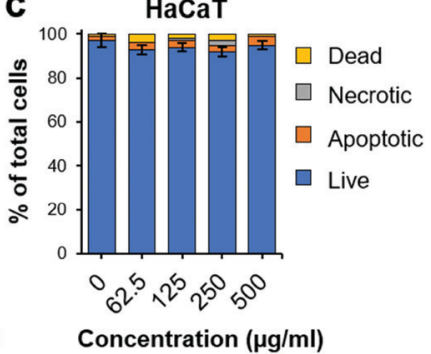

Fig. 6 (a) Representative fluorescence images of HDF and HaCaT counterstained with Hoechst 33342 (blue), Calcein AM (green) and PI (red) treated with $250 \mathrm{\mu g} \mathrm{ml}^{-1} \mathrm{NO}$-nanofrustules for 24 hours. Scale bar $=10$ $\mu \mathrm{m}$. Image cytometer based quantitative analysis of live/apoptotic cells for (b) HDF and (c) HaCaT. Experiments were triplicated. Data represent a mean \pm standard deviation $(n=5)$.

Consistently, for both cell types, the annexin V/PI cell viability assay results in Fig. $6 \mathrm{~b}$ show that more than $90 \%$ of the cells were viable, with less than $5 \%$ undergoing apoptosis, when treated with up to $500 \mu \mathrm{g} \mathrm{ml}^{-1}$ of NO-nanofrustules for 2 hours, which is within the time scale of antibacterial studies. The observations are in line with previous findings in the literature, as a similar dose of NO-releasing particles can be detrimental to bacteria but not mammalian cells due to sheer differences in size, uptake mechanism and membrane structures. $^{52}$

The results led us to conclude that NO-nanofrustules are cytocompatible with human dermal cells and shows great potential to be used for skin-related anti-infective therapies such as treatment of lesions in wounds and eczema. It is a proven strategy to use such nanomaterials under inflammatory conditions, whereby the nanomaterials can be absorbed on the surface of pathogenic microbes, not only disrupting any imminent biofilm formation but also blocking the active membrane fragments and adhesion factors, finally leading to their clearance from the wound. ${ }^{58}$

\section{Conclusions}

In summary, we developed novel diatom-inspired NO-releasing nanofrustules with high efficacy in eradicating Gram-positive $S$. aureus and Gram-negative E. coli bacteria. Besides showing the non-specific broad-spectrum lethality of the material, we also elucidated the mechanism of bacteria killing which leverages on the entropy driven aggregation behavior of the unique $2 \mathrm{D}$ porous architecture. The aggregation not only helps to capture and trap the bacteria in the process, but also creates a favorable environment for NO diffusive action. Importantly,

NO-nanofrustules were observed to elicit negligible cytotoxicity within our experimental conditions, suggesting that the platform is biocompatible for future clinical exploitation. In the broader context, our nano-enabled "capture-and-release" bactericidal strategy can be extended to design a range of multifunctional 2D nanomaterials to combat wound infections.

\section{Experimental}

\section{Materials}

The following materials were used without any further modifications, unless stated otherwise. Sulfuric acid $\left(\mathrm{H}_{2} \mathrm{SO}_{4}, 98 \%\right)$, phosphorus pentoxide $\left(\mathrm{P}_{2} \mathrm{O}_{5}\right)$, potassium permanganate $\left(\mathrm{KMnO}_{4}\right)$, sodium nitrate $\left(\mathrm{NaNO}_{3}\right)$, hydrogen peroxide $(30 \%$, $\mathrm{H}_{2} \mathrm{O}_{2}$ ), cetyltetraammonium bromide (CTAB), tetraethyl orthosilicate (TEOS), 3-mercaptopropyl trimethoxysilane (MPTMS), sodium nitrite $\left(\mathrm{NaNO}_{2}\right)$, sodium hydroxide $(\mathrm{NaOH})$ and $S$-nitroso- $n$-acetylpenicillamine (SNAP) were purchased from Sigma-Aldrich. Pentetic acid (DTPA) was purchased from Ark Pharm. Graphene nanopowder (0541DX) was purchased from SkySpring Nanomaterials. Anhydrous ethanol (99.9\%, EtOH) and anhydrous methanol $(99.9 \% \mathrm{MeOH})$ were purchased from Aik Moh Pte Ltd.

\section{Synthesis procedures}

Synthesis of GO nanosheets. $23 \mathrm{ml}$ of concentrated $\mathrm{H}_{2} \mathrm{SO}_{4}$ was added to a round-bottomed flask and kept in an ice bath $\left(0-5{ }^{\circ} \mathrm{C}\right) .1 \mathrm{~g}$ of $\mathrm{P}_{2} \mathrm{O}_{5}$ and $3 \mathrm{~g}$ of $\mathrm{KMnO}_{4}$ were slowly added to the flask immersed in ice under continuous stirring at $300 \mathrm{rpm}$ for 3 mins. $0.5 \mathrm{~g}$ of $\mathrm{NaNO}_{3}$ and $0.5 \mathrm{~g}$ of graphene nanopowder were mixed and finely ground using a mortar and pestle. The resultant powder was slowly added to the chilled mixture and stirred at room temperature. After 10 minutes, the solution temperature was increased to $35{ }^{\circ} \mathrm{C}$ and stirred for 1 hour. $10 \mathrm{ml}$ of deionized (DI) water (Millipore, Merck) was added dropwise to the flask via a $1 \mathrm{ml}$ pipette to avoid overheating the solution due to the resultant exothermic reaction. The solution's temperature was then adjusted to $85{ }^{\circ} \mathrm{C}$ for 15 minutes before cooling to room temperature. $5 \mathrm{ml}$ of $\mathrm{H}_{2} \mathrm{O}_{2}$ was added dropwise to the flask and a bright yellow coloration was observed indicating the presence of highly oxidized GO. To remove metal impurities, the flask's contents were washed thrice with $1 \mathrm{M} \mathrm{HCl}$ and twice with DI water. The resultant GO dispersion was then dialyzed in DI water using a cellulose membrane dialysis tube (10 kDa, Thermo Scientific) for at least 72 hours, changing the dialysate at least once every 12 hours. The neutralized, dialyzed solution was then centrifuged at $8500 \mathrm{rpm}$ for 5 minutes to increase the lateral size homogeneity of GO. Part of the dialysis product was then lyophilized for 24 hours to obtain solid GO nanoflakes.

One-pot synthesis of thiol functionalized (SH-)nanofrustules. This method was modified from a previously reported protocol. ${ }^{59}$ Briefly, a $0.28 \mathrm{M}$ CTAB solution was prepared by adding CTAB and $100 \mathrm{ml}$ of DI water to a round-bottomed flask. At $80{ }^{\circ} \mathrm{C}, 27 \mathrm{mg}$ of $\mathrm{GO}$ was added to the flask and the contents 
were stirred at $300 \mathrm{rpm}$ for 1 hour. The solution's $\mathrm{pH}$ was adjusted to 11.5 using $2 \mathrm{M}$ of $\mathrm{NaOH}$. $1.429 \mathrm{ml}$ of TEOS and $0.829 \mathrm{ml}$ of MPTMS were pre-mixed and then added dropwise to the solution. The reaction mixture was stirred at $80{ }^{\circ} \mathrm{C}$ and $500 \mathrm{rpm}$ for 2 hours, followed by centrifugation at $8000 \mathrm{rpm}$ for 5 minutes. The pellet obtained was washed twice using a solution containing equal parts by volume of $1 \mathrm{M}$ of $\mathrm{HCl}$ and $\mathrm{EtOH}$ ( $\mathrm{HCl}-\mathrm{EtOH})$ before incubating with the $\mathrm{HCl}-\mathrm{EtOH}$ mixture at $60{ }^{\circ} \mathrm{C}$ and stirred at $500 \mathrm{rpm}$. After 48 hours, the $\mathrm{SH}$-nanofrustules were washed thrice with EtOH before drying using a rotary evaporator $\left(50{ }^{\circ} \mathrm{C}\right.$, pressure $\left.60 \mathrm{mbar}\right)$ for at least 1 hour.

NO loading for SH-nanofrustules. $16 \mathrm{mg}$ of $\mathrm{SH}^{-}$ nanofrustules were added to $8 \mathrm{ml}$ of $\mathrm{MeOH}$ in a $50 \mathrm{ml}$ roundbottomed flask to make a methanolic mixture of $2 \mathrm{mg} \mathrm{ml}^{-1}$. $4 \mathrm{ml}$ of $5 \mathrm{M} \mathrm{HCl}$ was then added to the flask while the contents are stirred in an ice bath at $300 \mathrm{rpm} .4 \mathrm{ml}$ of a pre-chilled mixture consisting of $500 \mu \mathrm{M}$ DTPA and ten-fold molar excess of $\mathrm{NaNO}_{2}$ was added to the flask in the absence of light and incubated in an ice bath for 1 hour. The solution was washed thrice using pre-chilled $500 \mu \mathrm{M}$ DTPA and once with pre-chilled $\mathrm{MeOH}$. The solid pellet product was lyophilized for 30 minutes before storing in a $-40{ }^{\circ} \mathrm{C}$ freezer under dark conditions.

\section{Characterization methods}

Ellman assay for quantification of sulfhydryl groups. Briefly, a reaction buffer of $0.1 \mathrm{M}$ sodium phosphate, $\mathrm{pH}$ 8.0, containing $1 \mathrm{mM}$ EDTA was prepared. Then $4 \mathrm{mg} \mathrm{ml}^{-1}$ of the Ellman Reagent was dissolved in the reaction buffer. $150 \mu \mathrm{l}$ of the DTNB solution was then mixed with $50 \mu \mathrm{l}$ of SH-nanofrustules suspension $\left(50 \mu \mathrm{g} \mathrm{ml}^{-1}\right)$ in each well of a 96 -well plate. The absorbance was measured in a microplate reader at $405 \mathrm{~nm}$. Sample solutions with particles at desired concentrations were used as blanks. The concentration of sulfhydryl groups was then determined from a standard curve generated using different known concentrations of $\mathrm{N}$-acetyl cysteine.

NO release measurement with Griess assay. The assay was evaluated in physiological buffer (PBS, pH 7.4) at $37{ }^{\circ} \mathrm{C}$, DI water and $10 \%(\mathrm{v} / \mathrm{v})$ Luria-Bertani (LB) broth (Miller). The test was carried out in a 96-well plate with five replicates. Briefly, 125, 250 and $500 \mu \mathrm{g} \mathrm{ml} \mathrm{m}^{-1}$ of NO-nanofrustules in desired medium of suspension were prepared in a centrifuge tube with a total volume exceeding $1 \mathrm{ml}$. At designated time points, the suspension was centrifuged at $8000 \mathrm{rpm}$ for 3 minutes and $150 \mu \mathrm{l}$ of the supernatant was collected and added to the wells of a 96-well plate. Then, $20 \mu \mathrm{l}$ of the Griess reagent (Biotium) was added and the plate was incubated at RT in the dark for 10 minutes before measuring the absorbance at $548 \mathrm{~nm}$ with a spectrophotometer. Various concentrations of sodium nitrite solution were used as standards and a standard curve of absorbance against concentration was plotted.

Dynamic light scattering (DLS) and zeta potential measurements. Nanoparticle solutions were dispersed in DI water and sonicated to reduce polydispersity before a ZetaSizer Nano ZS (Malvern, Herrenberg, Germany) was used to measure the mean hydrodynamic radii and zeta potential of the studied nanoparticles. A minimum of five measurements for each sample were performed using a $633 \mathrm{~nm}$ He-Ne laser operating at an angle of $173^{\circ}$. The equipment uses the Stokes-Einstein equation and Henry correction to the Smoluchowski equation to obtain the hydrodynamic radius and zeta potential, respectively.

Nitrogen sorption measurement. The Brunauer-EmmettTeller (BET) surface area and Barrett-Joyner-Halenda (BJH) pore size distribution of SH-nanofrustules dry powder samples were determined using the Micromeritics ASAP TriStar 3020 Instrument with nitrogen gas adsorption and desorption. The degassing procedure was carried out at $60{ }^{\circ} \mathrm{C}$ overnight under vacuum conditions (100 mTorr).

Fourier-transform infrared spectroscopy (FTIR). Fourier Transform Infrared (FTIR) spectroscopy was used to determine chemical structures of the samples. A PerkinElmer Frontier FTIR spectrometer was used to scan the samples from 4000 to $400 \mathrm{~cm}^{-1}$ with potassium bromide $(\mathrm{KBr})$ as the background spectra. Prior to measurement, dry samples were mixed with $\mathrm{KBr}$ in a mass ratio of $1: 20$ and pressed to form disks.

Scanning electron microscopy (SEM) and energy dispersive X-ray spectroscopy (EDX). JEOL FESEM 6340F was used to investigate the surface morphology of the as-synthesized nanomaterials. Briefly, $0.5 \mathrm{mg}$ of the powdered materials mentioned above were dispersed in $1 \mathrm{ml}$ of absolute ethanol by sonication and $5 \mu \mathrm{l}$ of the dispersion was dropped on a freshly cleaved mica disc, dried in air overnight and sputter-coated with gold at $20 \mathrm{~mA}$ for $10 \mathrm{~s}$. Then, an operating voltage of $5 \mathrm{kV}$ was used to view the sample. For EDX experiments, the working distance and the operating voltage were increased to $15 \mathrm{~cm}$ and $10 \mathrm{kV}$, respectively. The average particle size of 10 individual particles in the SEM image was calculated after measurement using ImageJ software.

Transmission electron microscopy (TEM). Carl Zeiss Libra 100 TEM was used to examine the fabricated mesoporous structures and to determine pore size and particle size in greater detail. $50 \mu \mathrm{g} \mathrm{ml} \mathrm{m}^{-1}$ sample solutions were prepared by dispersing sample nanoparticles in $\mathrm{EtOH}$ and sonicating for 10 minutes. Next, $10 \mu \mathrm{l}$ of the solution was dropped onto a continuous carbon TEM grid (Latech Pte Ltd) and air-dried at $37{ }^{\circ} \mathrm{C}$ overnight. The mean particle size of 10 individual particles in the TEM image was calculated post-measurement using ImageJ software.

Broth and agar dilution assay for antibacterial testing. $E$. coli (ATCC 25922) and $S$. aureus (ATCC 25923) were cultured in $20 \mathrm{ml}$ of Luria-Bertani (LB) medium (Miller formulation) for 6 to 8 hours to reach the mid-exponential growth phase. The optical density (OD) at $600 \mathrm{~nm}$ of the bacterial suspension was measured using a spectrophotometer to estimate the number of bacteria commonly termed as the colony forming units (CFU) using a self-determined calibration curve. Briefly, OD600 = 1 was equivalent to $2.3 \times 10^{10}$ and $1.1 \times 10^{9} \mathrm{CFU}$ per $\mathrm{ml}$ for $S$. aureus and $E$. coli, respectively, post-calibration using the agar counting method. Next, a bacterial stock was prepared by serially diluting the bacterial suspension to $1 \times 10^{6} \mathrm{CFU}$ per $\mathrm{ml}$ using the fresh LB medium. Then, $90 \mu \mathrm{l}$ of the sample material 
suspension prepared at desired concentration was added to each well of a 96-well plate (Greiner Bio One) prior to adding $10 \mu \mathrm{l}$ of bacterial stock. The well plate was wrapped in foil to reduce evaporation and incubated at $37{ }^{\circ} \mathrm{C}$ in a static incubator for 2 hours or 24 hours. At designated time points, $10 \mu \mathrm{l}$ of the bacterial suspension was removed from the control well and wells of interest and diluted $10000 \times$ with fresh LB medium and plated on agar as a part of the agar dilution assay.

For the broth microdilution assay, $10 \mu \mathrm{l}$ of the PrestoBlue reagent (Invitrogen) was added to each well and incubated for 1 hour. Fluorescence readings were taken with a spectrophotometer at an excitation wavelength of $560 \mathrm{~nm}$ and an emission wavelength of $590 \mathrm{~nm}$; the bacterial cell viability was calculated as a percentage of fluorescence intensity $I$ of the sample compared to that of the negative control in eqn (1).

$$
\text { Bacterial viability }(\%)=\frac{I_{\text {sample }}-I_{\text {blank }}}{I_{\text {control }}-I_{\text {blank }}}
$$

Antimicrobial activity was expressed as the minimum inhibitory concentration (MIC), i.e., the lowest concentration that did not result in the change in color of the PrestoBlue after 2 hours of incubation. In addition, the MIC in the agar dilution assay can be defined as the lowest concentration which did not result in observable bacterial growth in agar after 2 hours. Although MIC is usually determined based on turbidity changes, the presence of opaque nanoparticles may complicate the measurement of turbidity. Therefore, assays based on optical density (OD) and turbidity were not employed in this work.

Cytotoxicity assays. Human dermal fibroblasts (HDF) and human immortalized keratinocytes (HaCaT) were cultured in Dulbecco's Modified Eagle Medium (DMEM) with 10\% fetal bovine serum at $37{ }^{\circ} \mathrm{C}$ and $5 \% \mathrm{CO}_{2}$. For metabolic activity assessment, the cells were cultured in a 96 well plate. NOnanofrustules suspended in DMEM were added to the cells when the cells reached $50 \%$ confluency, followed by further incubation for 24 hours. $10 \mu \mathrm{l}$ of Presto Blue was added to each well and the fluorescence at 560/590 nm was analysed using a spectrophotometer after 1 hour. For the cellular LIVE/DEAD assay, the cells are cultured in Lab-Tek ${ }^{\mathrm{TM}}$ chamber slides and stained with Calcein AM for live, propidium iodide (PI) for dead, and Hoechst dye for nuclei, according to standard protocols. The fluorescence intensities of the micrographs were analyzed using ImageJ software. For apoptotic assays, the cells were cultured in 12 well plates and trypsinized before resuspending in binding buffer. The Annexin V Alexa Fluor 488 conjugate and PI were then added into the cell suspension and the results were analyzed using the Arthur NanoEntek ${ }^{\mathrm{TM}}$ automated cell counter after 15 minutes of incubation at room temperature.

Statistical analysis. Statistical analysis of experimental data was conducted using Microsoft Excel with RealStats macro for the Mann-Whitney non-parametric test for statistical significance (for samples with $n=3$ ) or the Student's $t$-test with unequal variances (for samples with $n$ larger than 3 ). Plotted graphs contain the mean and standard deviation of at least 3 independent samples from the same experiment or 3 separate experiments.

\section{Conflicts of interest}

There are no conflicts to declare.

\section{Acknowledgements}

The authors gratefully acknowledge support from the Singapore's Ministry of Education (MOE) Academic Research Fund Tier 1 (RG38/20 2020-T1-001-152) and the Nanyang Technological University (NTU)-Harvard School of Public Health (HSPH) Initiative for Sustainable Nanotechnology Program (NTU-HSPH 18002). The authors would also like to acknowledge the Facility for Analysis, Characterization, Testing and Simulation (FACTS), Nanyang Technological University, Singapore, for the use of their electron microscopy facilities.

\section{References}

1 S. M. Diene and J. M. Rolain, Clin. Microbiol. Infect., 2014, 20, 831-838.

2 L. S. J. Roope, R. D. Smith, K. B. Pouwels, J. Buchanan, L. Abel, P. Eibich, C. C. Butler, P. S. Tan, A. S. Walker, J. V. Robotham and S. Wordsworth, Science, 2019, 364.

3 D. Ferber, Science, 2000, 288, 792-794.

4 G. Zhu, Z. Sun, P. Hui, W. Chen and X. Jiang, ACS Biomater. Sci. Eng., 2020, 7, 1827-1835.

5 Y. Zhu, M. Ramasamy and D. K. Yi, ACS Appl. Mater. Interfaces, 2014, 6, 15078-15085.

6 A. C. Ferrari, F. Bonaccorso, V. Fal'ko, K. S. Novoselov, S. Roche, P. Bøggild, S. Borini, F. H. L. Koppens, V. Palermo and N. Pugno, et al., Nanoscale, 2015, 7, 4598-4810.

7 G. Sai-Anand, A. Sivanesan, M. R. Benzigar, G. Singh, A.-I. Gopalan, A. V. Baskar, H. Ilbeygi, K. Ramadass, V. Kambala and A. Vinu, Bull. Chem. Soc. Jpn., 2019, 92, 216-244.

8 L. Wang, C. Hu and L. Shao, Int. J. Nanomed., 2017, 12, 1227-1249.

9 S. Liu, T. H. Zeng, M. Hofmann, E. Burcombe, J. Wei, R. Jiang, J. Kong and Y. Chen, ACS Nano, 2011, 5, 6971-6980.

10 T. Sreeprasad, M. S. Maliyekkal, K. Deepti, K. Chaudhari, P. L. Xavier and T. Pradeep, ACS Appl. Mater. Interfaces, 2011, 3, 2643-2654.

11 T. I. Kim, B. Kwon, J. Yoon, I. J. Park, G. S. Bang, Y. Park, Y. S. Seo and S. Y. Choi, ACS Appl. Mater. Interfaces, 2017, 9, 7908-7917.

12 R. P. Pandey, P. A. Rasheed, T. Gomez, K. Rasool, J. Ponraj, K. Prenger, M. Naguib and K. A. Mahmoud, ACS Appl. Nano Mater., 2020, 3, 11372-11382.

13 H. Zhao, C. Zhang, Y. Wang, W. Chen and P. J. J. Alvarez, Environ. Sci. Technol., 2018, 52, 12783-12791.

14 F. Perreault, A. F. de Faria, S. Nejati and M. Elimelech, ACS Nano, 2015, 9, 7226-7236. 
15 W. Lu, K. Yao, J. Wang and J. Yuan, J. Colloid Interface Sci., 2015, 437, 35-41.

16 K. H. Tan, S. Sattari, S. Beyranvand, A. Faghani, K. Ludwig, K. Schwibbert, C. Böttcher, R. Haag and M. Adeli, Langmuir, 2019, 35, 4736-4746.

17 B. Ahmed, B. Solanki, A. Zaidi, M. S. Khan and J. Musarrat, Toxicol. Res., 2019, 8, 246-261.

18 S. Fulaz, S. Vitale, L. Quinn and E. Casey, Trends Microbiol., 2019, 27, 915-926.

19 T. M. Mohona, A. Gupta, A. Masud, S.-C. Chien, L.-C. Lin, P. C. Nalam and N. Aich, Environ. Sci. Technol., 2019, 53, 4161-4172.

20 S. Su, J. Wang, E. Vargas, J. Wei, R. Martínez-Zaguilán, S. R. Sennoune, M. L. Pantoya, S. Wang, J. Chaudhuri and J. Qiu, ACS Biomater. Sci. Eng., 2016, 2, 1357-1366.

21 A. Panáček, L. Kvítek, M. Smékalová, R. Večeřová, M. Kolář, M. Röderová, F. Dyčka, M. Šebela, R. Prucek, O. Tomanec and R. Zbořil, Nat. Nanotechnol., 2018, 13, 65-71.

22 E. de Alteriis, V. Maselli, A. Falanga, S. Galdiero, F. M. Di Lella, R. Gesuele, M. Guida and E. Galdiero, Infect. Drug Resist., 2018, 11, 915.

23 J. Xue, Y. Niu, M. Gong, R. Shi, D. Chen, L. Zhang and Y. Lvov, ACS Nano, 2015, 9, 1600-1612.

24 F. Han, S. Lv, Z. Li, L. Jin, B. Fan, J. Zhang, R. Zhang, X. Zhang, L. Han and J. Li, NPG Asia Mater., 2020, 12, 1-11.

25 S. R. Ali, S. Pandit and M. De, ACS Appl. Bio Mater., 2018, 1, 967-974.

26 K. T. Nguyen, Z. Wu, T. Huang and C. Y. Tay, Curr. Med. Chem., 2018, 25, 1420-1432.

27 F. C. Fang, J. Clin. Invest., 1997, 99, 2818-2825.

28 K. E. A. Darling and T. J. Evans, Infect. Immun., 2003, 71, 2341-2349.

29 V. F. Cardozo, C. A. C. Lancheros, A. M. Narciso, E. C. S. Valereto, R. K. T. Kobayashi, A. B. Seabra and G. Nakazato, Int. J. Pharm., 2014, 473, 20-29.

30 G. Narin, Ç. B. Albayrak and S. Ülkü, Appl. Clay Sci., 2010, 50, 560-568.

31 M. R. Mihu, U. Sandkovsky, G. Han, J. M. Friedman, J. D. Nosanchuk and L. R. Martinez, Virulence, 2010, 1, 62-67.

32 E. M. Hetrick, J. H. Shin, H. S. Paul and M. H. Schoenfisch, Biomaterials, 2009, 30, 2782-2789.

33 L. R. Martinez, G. Han, M. Chacko, M. R. Mihu, M. Jacobson, P. Gialanella, A. J. Friedman, J. D. Nosanchuk and J. M. Friedman, J. Invest. Dermatol., 2009, 129, 2463-2469.

34 R. B. Weller, J. Invest. Dermatol., 2009, 129, 2335-2337.

35 N. Kröger, Curr. Opin. Chem. Biol., 2007, 11, 662-669.

36 J. T. Chao, M. J. P. Biggs and A. S. Pandit, Expert Opin. Drug Delivery, 2014, 11, 1687-1695.
37 F. Zou, H. Zhou, D. Y. Jeong, J. Kwon, S. U. Eom, T. J. Park, S. W. Hong and J. Lee, ACS Appl. Mater. Interfaces, 2017, 9, 1343-1351.

38 Y. Wang, Y. Yang, Y. Shi, H. Song and C. Yu, Adv. Mater., 2020, 32, 1904106.

39 C. H. Chen, S. Hu, J. F. Shih, C. Y. Yang, Y. W. Luo, R. H. Jhang, C. M. Chiang and Y. Hung, Sci. Rep., 2017, 7, 3908.

40 D. A. Riccio, J. L. Nugent and M. H. Schoenfisch, Chem. Mater., 2011, 23, 1727-1735.

41 M. Mishra, A. P. Arukha, T. Bashir, D. Yadav and G. B. K. S. Prasad, Front. Microbiol., 2017, 8, 1239.

42 L. Li, X. L. Liu, H. Y. Geng, B. Hu, G. W. Song and Z. S. Xu, J. Mater. Chem. A, 2013, 1, 10292-10299.

43 M. Szekeres, J. Tóth and I. Dékány, Langmuir, 2002, 18, 2678-2685.

44 K. T. Nguyen, S. Sreejith, J. Joseph, T. He, P. Borah, E. Y. Guan, S. W. Lye, H. Sun and Y. Zhao, Part. Part. Syst. Charact., 2014, 31, 1060-1066.

45 M. J. Malone-Povolny and M. H. Schoenfisch, ACS Appl. Mater. Interfaces, 2019, 11, 12216-12223.

46 J. O. Kim, J.-K. Noh, R. K. Thapa, N. Hasan, M. Choi, J. H. Kim, J.-H. Lee, S. K. Ku and J.-W. Yoo, Int. J. Biol. Macromol., 2015, 79, 217-225.

47 P. L. Ritger and N. A. Peppas, J. Controlled Release, 1987, 5, 23-36.

48 Y. Lu, D. L. Slomberg, B. Sun and M. H. Schoenfisch, Small, 2013, 9, 2189-2198.

49 B. M. Grommersch, J. Pant, S. P. Hopkins, M. J. Goudie and H. Handa, ACS Appl. Mater. Interfaces, 2018, 10, 2291-2301.

50 T. Trunk, H. S. Khalil and J. C. Leo, AIMS Microbiol., 2018, 4, 140-164.

51 J. Pant, J. Gao, M. J. Goudie, S. P. Hopkins, J. Locklin and H. Handa, Acta Biomater., 2017, 58, 421-431.

52 H. Jeong, J.-H. Park, J. H. Shin, J.-C. Yoo, C. Y. Park and J. Hong, Chem. Mater., 2018, 30, 8528-8537.

53 O. Akhavan and E. Ghaderi, ACS Nano, 2010, 4, 5731-5736. 54 A. Vakurov, R. Brydson and A. Nelson, Langmuir, 2012, 28, 1246-1255.

55 C. Spengler, F. Nolle, J. Mischo, T. Faidt, S. Grandthyll, N. Thewes, M. Koch, F. Müller, M. Bischoff, M. A. Klatt and K. Jacobs, Nanoscale, 2019, 11, 19713-19722.

56 H. Song, Y. Ahmad Nor, M. Yu, Y. Yang, J. Zhang, H. Zhang, C. Xu, N. Mitter and C. Yu, J. Am. Chem. Soc., 2016, 138, 6455-6462.

57 J. Chen, H. Peng, X. Wang, F. Shao, Z. Yuan and H. Han, Nanoscale, 2014, 6, 1879-1889.

58 S. Lavie and G. Stotzky, Appl. Environ. Microbiol., 1986, 51, 65-73. 59 Z.-M. Wang, W. Peng, Y. Takenaka, N. Yoshizawa, K. Kosuge, W. Wang and G. A. Ozin, Adv. Funct. Mater., 2017, 27, 1704066. 